\title{
The Design and First Results of a Dedicated Corrector (S)TEM.
}

\author{
M. A. vd Stam, P. Tiemeijer, B. Freitag, M. Stekelenburg, and J. Ringnalda \\ FEI Company, Building AAE, Achtseweg Noord 5, Eindhoven, The Netherlands
}

In all TEM imaging, the spatial resolution is predominantly limited by the spherical aberration and chromatic aberration of the objective lens $[1,2]$. These aberrations cause the information in the image to be blurred. This information can be retrieved by through-focus series reconstruction or by holography. Alternatively, a more direct way is to correct the spherical aberration by incorporating a Cs corrector in the TEM column [3], thus making the point resolution equal to the information limit. For the situation where the aberrations are corrected on the image (Objective lens correction), a system shows enhancement of the resolution all the way down to the information limit. For the situation where the aberrations are corrected on the probe [4] (Condenser lens correction), the probe size can be improved however system stability starts to play a more and more important role in determining the final performance of the total system

At FEI Company, the theory of improving the resolution by combining a monochromator [5] and a Cs corrector was successfully proven on a Tecnai F20ST [6]. It was demonstrated that directly interpretable resolutions below 1 Ångström were obtained for the first time, which is significantly better than any TEM operating at 200KV had ever reached before. In order to reach the next level of performance needed for corrected microscopy, FEI has executed an intensive R\&D program over the last 4 years to arrive at an S/TEM dedicated for correctors. By design, the following additional aspects were addressed: ultimate stability and ultimate flexibility. The new corrected era calls for new rules, a new microscope as well as new results.

Whereas the objective lens used to be the most important aspect in terms of resolution and tilt capabilities, this requirement no longer plays a significant role in corrected systems. Since a corrector allows a system to obtain directly interpretable resolution down to the information limit of the total microscope system, the value for information limit becomes the resolution-limiting specification in imaging, and therefore a key specification of the base microscope system. For enhanced probe and analysis performance, total stability of the complete system, both electronically and mechanically, will determine the performance. It is for this reason that FEI has built a new platform especially for corrected optics (correctors and monochromator) that operates up to $300 \mathrm{kV}$.

The new (S)TEM corrector platform is a revolutionary new design with ConstantPower ${ }^{\mathrm{TM}}$ lenses and stress-free electron optical lens modules. All cross-talk and hysteresis issues which exist in conventional columns have been significantly reduced or eliminated by this new design. A better mechanical alignment of the system is designed into the new column, allowing the range of deflectors etc. to be reduced. The very flexible high tension circuitry will enable users to select the optimum high voltage between 80 to $300 \mathrm{kV}$; since some materials damage more at $300 \mathrm{kV}$, some damage less. The Cs correctors can be tuned at different voltages between $80 \mathrm{kV}$ to $300 \mathrm{kV}$ through a very straightforward SW user interface. Once tuned these values are obviously stored in software for future use. The switching of modes, magnifications, and techniques is fast and reproducible due to short stabilization time needed. 
The addition of a third full condenser lens now means that with a monochromator, even when selecting the high resolution energy slit, the spatial resolution of the system will be less than 1 Ångström in combination with a Cs corrector on the probe. With this extra condenser lens, the flexibility now exists to vary the convergence angle while keeping the spot focused; also setting up extremely small but narrow parallel beams is trivial, as is keeping the illumination perfectly parallel while changing the exposed area of the sample. While more results will be presented at the conference itself, some important early achievements are shown in Fig. 1. which shows images of the information limit obtained with this system, as fitted with a S-TWIN lens and no correctors or monochromator. It is clear that the information goes well past the 1 Ångström mark. In a line resolution experiment, as can be seen in Fig. 2, spots in the FFT can be seen approaching $0.05 \mathrm{~nm}$ indicating superb stability.

\section{References}

[1] Knoll, M. \& Ruska, E. Das Elektronenmikroskop. Z. Physik 78, 318-339 (1932).

[2] Scherzer, O. Über einige Fehler von Elektronenlinsen. Z. Physik 101, 593-603 (1936).

[3] M. Haider, G. Braunhausen, and E. Schwan, Optik 99 (1995) 167.

[4] Batson, P. E., Dellby, N. \& Krivanek, O. L. Nature 418, 617-620 (2002).

[5] P.C. Tiemeijer, Inst. Phys. Conf. Ser. 161 (1999) 191

[6] B. Freitag et al., Ultramicroscopy 102 (2005) 209.

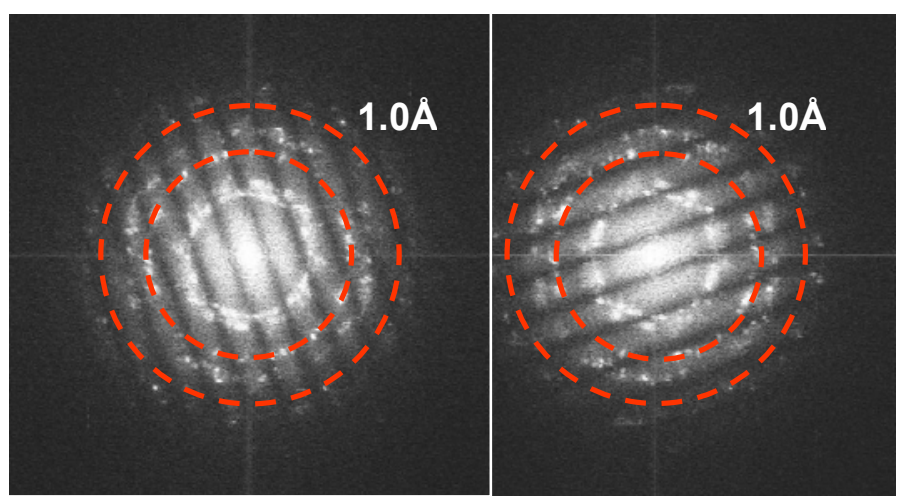

Fig. 1. Young's fringes in FFT showing better than 1 Ångström transfer in information on new dedicated corrector S/TEM system, even without corrector or monochromator fitted to it.

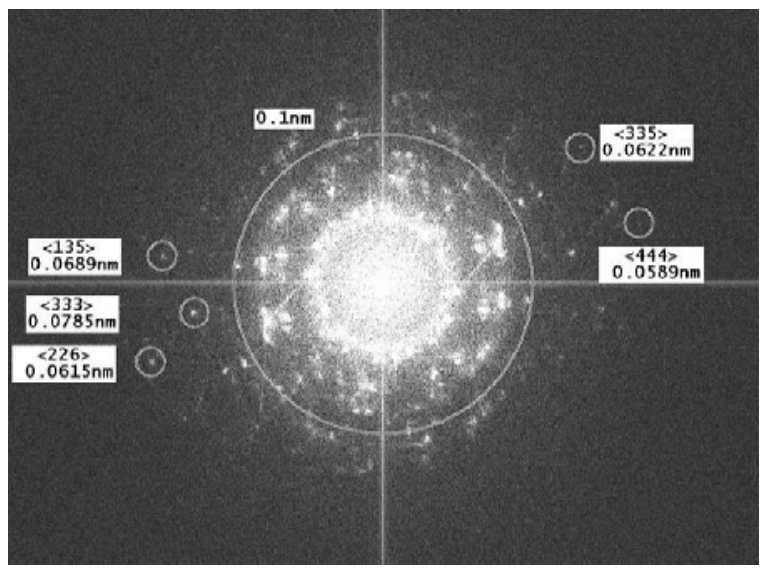

Fig 2: FFT of a high resolution image of gold with the reflections indicated. 